\title{
LA EDUCACIÓN AMBIENTAL EN LA FORMACIÓN DOCENTE INICIAL EN MÉXICO
}

\author{
Raúl Calixto Flores, Mayra García Ruiz, Jessica Gloria Rocío del Socorro Rayas Prince ${ }^{1}$
}

\begin{abstract}
Resumen
El artículo realiza una revisión teórica de la educación ambiental en la formación docente inicial en el nivel medio básico, a partir del análisis del plan de estudios de la educación secundaria y el plan de estudios para la formación docente de México. La educación ambiental en el nivel de secundaria se encuentra como contenido transversal, sin embargo, la transversalidad está ausente en la formación docente inicial. Se encuentran pocos contenidos, principalmente en las licenciaturas de Biología, Geografía y Química. La educación ambiental es uno de los temas pendientes de la formación docente. Los futuros profesores requieren de conocimientos, actitudes y valores que les permitan incidir significativamente en la formación ambiental de los jóvenes estudiantes.
\end{abstract}

Palabras clave: Educación ambiental. Educación secundaria. Formación docente. Plan de estudios.

\section{ENVIRONMENTAL EDUCATION IN INITIAL TEACHER TRAINING IN MEXICO}

\begin{abstract}
This article presents a theoretical review on environmental education in initial teacher training in the basic secondary level based on the analysis of the secondary education and teacher training curricula in Mexico. Environmental education at the secondary level is considered a crosscutting content; however, crosscutting is absent in initial teacher training. Regarding the training of future teachers, there are few contents, mainly in biology, geography and chemistry. Environmental education is one of the issues lacking in the training of teachers, who require knowledge, attitudes and values that enable them to have a significant impact on the environmental education of young students.
\end{abstract}

Keywords: Environmental education. Secondary education. Teacher training. Curriculum.

\section{EDUCAÇÃO AMBIENTAL NA FORMAÇÃO INICIAL DE PROFESSORES NO MÉXICO}

\section{Resumo}

Este artigo apresenta uma revisão teórica sobre educação ambiental na formação inicial de professores no nível secundário com base na análise dos currículos do ensino secundário e da formação de professores no México. A educação ambiental no nível secundário é encontrada como conteúdo transversal, mas a transversalidade está ausente no treinamento inicial de professores. No que se refere ao treinamento de futuros professores, há poucos conteúdos, principalmente nos bacharelados em Biologia, Geografia e Química. Percebe-se que a educação

\footnotetext{
${ }^{1}$ Professores-pesquisadores da Universidad Pedagógica Nacional - México
} 
ambiental é uma das questões pendentes de treinamento inicial de professores, que necessitam conhecimentos, atitudes e valores que lhes permitam impactar de forma significativa na educação ambiental dos jovens estudantes.

Palavras-chave: Educação ambiental. Ensino secundário. Formação de professores. Currículo.

\section{Introducción}

La educación ambiental está presente tanto en los ámbitos escolarizados como en los no. En cuanto al ámbito no escolarizado, constituye un espacio de diálogos abiertos, donde emergen proposiciones novedosas de integración y articulación para audiencias amplias (GASPARETTO; ZATTONI; PROTTI, 2012) en donde se pueden planificar e instrumentar estrategias flexibles e novedosas. En cuanto al ámbito escolar, el profesor es el principal agente educativo al planear, instrumentar y evaluar los procesos de enseñanza y aprendizaje. Los docentes desempeñan un papel fundamental para mediar entre los aprendizajes de los individuos y el medio ambiente natural y social (MATOS, 2009).

Barber y Mourshed (2007) identifican la formación de profesores como uno de los principales factores para mejorar los resultados de la educación. Un profesorado con una visión clara de las finalidades de su quehacer docente, con el conocimiento de los contenidos disciplinarios, de los procesos pedagógicos, de las etapas de aprendizaje de sus estudiantes y con una conciencia clara del compromiso social de su tarea, sin duda son factores claves para lograr los objetivos de los diferentes niveles del sistema educativo. Alcira y Perales (2006) consideran fundamental que los docentes en formación desnaturalicen los problemas ambientales e identifiquen su componente antropogénico. Para ello, proponen que se reconozcan "problemas de la realidad" (situaciones ambientales locales) y se busquen fundamentos y criterios para abordarlos en su comprensión con la diversidad de conocimientos disponibles.

Sin en el plan de estudios de la formación inicial de los profesores en México, se permea un interés técnico del conocimiento vinculado con la eficiencia en la obtención de resultados, el dominio de los contenidos científicos y el logro de los rasgos deseables del nuevo docente, incrementando así los "niveles de calidad". Al respecto, Castañeda (2010) advierte que en la formación en educación ambiental es necesario desarrollar herramientas conceptuales y metodológicas para la intervención, generando actitudes positivas hacia el ambiente. Sin embargo, en los cursos de la formación general y la formación común de las distintas licenciaturas, no se alude al medio ambiente, ni a la educación ambiental.

Se minimiza la importancia de la educación ambiental, sin considerar que los profesores en formación, a través de esta educación, logran sensibilizarse ante los problemas ambientales y desarrollan una conciencia de la responsabilidad que la sociedad, las instituciones y los individuos poseen en ello, Perales (2011).

En este artículo se aborda la educación ambiental en la formación docente inicial en el nivel medio básico, a partir de la revisión teórica del plan de estudios de la educación secundaria de la Reforma Integral de Educación Básica (RIEB) y el plan de estudios para la formación de profesores en educación secundaria de la Reforma Curricular de la Educación Normal (RCEN). 


\section{Educación ambiental}

El objeto de estudio de la educación ambiental se encuentra en las relaciones entre los seres humanos, entre estos y la sociedad, y la sociedad con la naturaleza. Los objetivos de la educación ambiental son múltiples, entre otros se encuentran:

- la formación de una conciencia sustentable;

- la generación de actitudes para la adaptación a los cambios ambientales;

- la construcción de respuestas novedosas ante los efectos de las alteraciones al medio ambiente;

- la elaboración de propuestas que incidan en la transformación de las relaciones de los seres humanos con el medio ambiente, con base en la sensibilización, en la alfabetización, en la información, en los conocimientos, en las actitudes y en los valores;

- el desarrollo de una conciencia sustentable y planetaria.

La educación ambiental implica el análisis crítico de las situaciones que se presentan en el medio ambiente; en este sentido se relaciona con el desarrollo de una perspectiva crítica y compleja que analiza la realidad desde las condiciones de diversos subsistemas interrelacionados.

La educación ambiental crítica se fundamenta en la pedagogía crítica, por tratarse, de acuerdo a Guimarães y Alvez (2012), de una propuesta libertadora y comprometida con la transformación y la resignificación de los valores individualistas, como una posibilidad de superar la educación ambiental vigente. La perspectiva crítica en la educación ambiental es necesaria, para lograr la generación de comportamientos solidarios, reflexivos, participativos y comprometidos con el bien común; así mismo de la perspectiva compleja, también es fundamental, como medio para promover una visión que reconozca la integración de las acciones locales, regionales, nacionales y globales, desde distintos ámbitos, como el educativo, así como desde diversas disciplinas.

En este sentido, las cuestiones ambientales remiten a problemáticas complejas que requieren de ser atendidas y estudiadas desde diversas disciplinas, ya que involucran diferentes ámbitos como el económico, el productivo, el físico - biológico entre otros los cuales conforman una totalidad con la agrupación de múltiples procesos que configuran un sistema complejo (GARCÍA, 2013).

Los problemas ambientales se multiplican, a diferencia de hace algunas décadas, la humanidad se acerca al punto de no retorno, es decir, las alteraciones ambientales están dando lugar a efectos irreversibles en el planeta que trastocan los ciclos fisicoquímicos y atmosféricos, que alteran los climas y ponen en riesgo la supervivencia de miles de maneras de vida. Al respecto, Lovelock (2007) menciona que el planeta ya se encuentra en una situación tal, que todos los esfuerzos que se realicen por reducir los efectos del cambio climático, no tendrán éxito. Por otra parte, Randers (2012) sostiene que en la segunda mitad del siglo XXI, el planeta tendrá una crisis ambiental severa, las vías sustentables ya no estarán al alcance de la humanidad.

Las condiciones del medio ambiente no solo se están alterando, sino que se están perturbando a un ritmo acelerado, con transformaciones diversas ocurriendo a la vez, las cuales no permiten adaptaciones progresivas ni de los procesos naturales, ni de los seres humanos; cada vez más se están alterando las condiciones esenciales para la vida y el cambio climático provoca también todo tipo de modificaciones como las biológicas y las geográficas en el planeta (DE AMBROSIO, 2014, 55). 
En el V Informe, el Panel Intergubernamental de Cambio Climático, advierte que cada uno de los tres últimos decenios ha sido sucesivamente más cálido en la superficie de la Tierra que cualquier decenio anterior desde 1850; que las consecuencias del cambio climático cambiarán la temperatura y los patrones de precipitación; señala un creciente riesgo de pérdida de ecosistemas y biodiversidad acuáticos terrestres y continentales, como también de los bienes, funciones y servicios ecosistémicos; y prevé que el cambio climático ocasionará un incremento de mala salud en muchas regiones y especialmente en los países en desarrollo de bajos ingresos, entre otras cuestiones más (Grupo Intergubernamental De Expertos Sobre El Cambio Climático, 2013). Las implicaciones ambientales del cambio climático en el medio ambiente, por lo general, no son abordadas de manera suficiente en la educación ambiental escolar.

La educación ambiental puede contribuir a la construcción de diversas estrategias, que favorezcan el desarrollo de estilos de vida sustentable que apoyen la integridad ecológica, la justicia social y económica, los modos de vida sustentables y el respeto a toda manera de vida; aprender a prevenir y resolver conflictos, respetar la diversidad cultural, crear una sociedad con un profundo respeto al cuidado y una vida en paz; aprender de los pueblos indígenas y los patrones tradicionales de vida que respetan y honran la Tierra y sus sistemas de apoyo a la vida; adaptar esta sabiduría a nuestro mundo que cambia rápidamente; hacer escogencias individuales, comunitarias, nacionales y hasta globales con la debida consideración hacia el bien colectivo (UNESCO, 2007).

En este sentido, González Gaudiano (1997, 1998, 2002, 2011) ha documentado el origen y el desarrollo de la educación ambiental en México. Este autor menciona que la educación ambiental desde su origen se le ha vinculado con las ciencias naturales proporcionando un sesgo ecológico. Para González Gaudiano, en la década de los noventa del siglo anterior cuando la educación ambiental comienza a ser reconocida en México como una cuestión de primer orden de manera más integral, ya que en las dos décadas anteriores la educación ambiental, se remitía solo a algunos sectores especializados en el tema. González Gaudiano (2011) señala que:

La educación ambiental para la sustentabilidad se ha posicionado dentro de la Secretaría de Educación Pública. Hoy, nadie discute como antaño sobre la importancia de darle a este campo una presencia creciente en planes y programas de estudio en todos los niveles y modalidades educativas. Y se reconoce que la formación docente en este campo es de una alta prioridad (GONZÁLEZ GAUDIANO, 2011, sp).

Esta importancia, a la que alude González Gaudiano con respecto a la formación docente, la Secretaria de Educación Pública (SEP) la aborda solamente en el discurso, porque son escasas las acciones; en este contexto emerge la importancia de realizar una revisión de la formación docente inicial en educación ambiental.

En México, la educación ambiental se ha incluido en todos los niveles educativos (SEMARNAT, 2006); incorporado contenidos ambientales en preescolar, primaria, secundaria, media superior, y en numerosas licenciaturas en educación superior; así como en las escuelas formadoras de docentes. La educación ambiental requiere formar profesores críticos de la realidad socio-ambiental, y participativos en la búsqueda de alternativas educativas. Se requiere de una: ...formación del profesorado más sensibilizado, capacitándolos para poder trabajar en grupos interdisciplinares que elaboren proyectos de educación ambiental que aglutinen e integren distintas disciplinas del currículum. Estos proyectos, no deben suponer nuevas incorporaciones de contenidos, sino un modo distinto de tratarlos (GARCÍA, 2007, 329). 
Desde la inclusión de la educación ambiental en el currículum escolar, se han generado propuestas para la formación de docentes; ya que esta formación es fundamental para que los profesores desarrollen las estrategias didácticas requeridas, puesto que se reconoce que los profesores pueden incidir de alguna manera en estas cuestiones desde sus acciones.

La revisión teórica que se realiza en el artículo, considera algunos de los principales aspectos de la educación ambiental, como la transversalidad, el origen de la problemática ambiental, la concepción del conocimiento, la concepción del medio ambiente y los contenidos de la educación ambiental. Primero, se analiza el plan de estudios del nivel medio básico (2011) y después el plan de formación de docentes en educación secundaria (2012).

\section{Educación ambiental en el nivel medio básico}

La educación secundaria o nivel medio básico constituye el último nivel de la educación básica en México; en el ciclo escolar 2013-2014, en este nivel educativo, de acuerdo a los datos de la SEP (2014), la población escolar ascendió a 6571858 de alumnos. Una gran proporción de estos jóvenes, ya no continuarán con sus estudios en los subsecuentes niveles educativos. En este mismo ciclo escolar, los alumnos inscritos en el nivel medio superior fue de 4682 336. De acuerdo a esta información, 1889522 de jóvenes desertaron del sistema escolar en su tránsito hacia la educación media superior. Esta situación, hace que la educación secundaria sea la última etapa escolar, en donde un amplio sector de la población puede acceder a la educación ambiental.

Plan de estudios 2011. La RIEB tiene sus antecedentes en los acuerdos secretariales emitidos por la SEP en el acuerdo número 348, por el que se determina el Programa de Educación Preescolar. El acuerdo número 540, por el que se establece el nuevo Plan y programas de estudio para educación primaria y el acuerdo número 384, por el que se establece el nuevo Plan y programas de estudio para la educación secundaria publicados, respectivamente, en el Diario Oficial de la Federación el 27 de octubre de 2004, el 26 de mayo de 2006 y el 20 de agosto de 2010. A partir de estos acuerdos, se inicia el proceso de revisión de los planes de estudio y del conjunto de programas de los niveles de preescolar, primaria y secundaria.

En educación secundaria en el ciclo escolar 2005-2006, se inició la prueba piloto de nuevos programas, y es hasta el ciclo escolar 2011-2012, cuando se inicia el nuevo plan de estudios y programas.

Para la implementación de la RIEB se tuvo que trabajar tanto con los problemas convencionales de orden institucional como con una negociación política, ya que este nivel como tal, presenta serios problemas de cobertura, de condiciones laborales del magisterio entre otras; lo que propició dificultades en la implementación de la reforma curricular sobre todo en cuestiones emergentes como la educación ambiental.

Aunque se planteaba a través de la reforma el fortalecimiento del enfoque y contenidos orientados por el desarrollo de competencias para integrar conocimientos, valores, actitudes y habilidades de manera compartida en todas las asignaturas, esto implicó diferentes retos, tanto en la organización escolar, como en el trabajo de los profesores en las diversas asignaturas. Aunque se proponía un trabajo menos fragmentado, se siguió dando prioridad a las asignaturas de Español, Matemáticas y Ciencias (MIRANDA, 2012).

Transversalidad. En la RIEB, la educación ambiental tiene un papel relevante al ser considerada como un campo transversal. Se establece desde el nivel de educación preescolar seis campos formativos, uno de éstos corresponde al de "exploración y conocimiento del mundo" 
considerando al mundo natural, cultura y vida social. En el acuerdo 384, se conceptualiza como: ...contenido transversal que articula los contenidos de las asignaturas en los tres niveles educativos. La intención es promover conocimientos, habilidades, valores y actitudes para que los estudiantes participen individual y colectivamente en el análisis, la prevención y la reducción de problemas ambientales, y favorecer así la calidad de vida de las generaciones presentes y futuras (SEP, 2006, $31)$.

La educación ambiental como "campo" o "contenido" transversal requiere, necesariamente, de docentes que puedan incorporar en su práctica, temas y contenidos relacionados con el conocimiento de los problemas ambientales y del cuidado del medio ambiente.

Origen de la problemática ambiental. La educación ambiental en el plan de estudios de educación secundaria, se orienta a fomentar la participación reflexiva del alumno ante los problemas ambientales, los cuales constituyen una realidad compleja que, necesariamente, debe ser abordada desde todas sus dimensiones. Entre los principios pedagógicos de la educación básica, señalado en el plan de estudios, se hace referencia a la necesidad de incorporar temas de relevancia social para que los estudiantes aprendan actuar con responsabilidad ante el medio natural y social.

Concepción del conocimiento. En la RIEB se proponen una serie de rasgos deseables para el estudiante como resultado del proceso de formación a lo largo de la educación básica, entre los que se encuentran, el referido a la promoción del cuidado de la salud y del ambiente como condiciones que favorecen un estilo de vida activo y saludable. Para lograrlo, se proponen el campos de formación "Exploración y comprensión del mundo natural y social", en el que se observa el predominio de los conocimientos disciplinarios relacionados con aspectos biológicos, históricos, sociales, políticos, económicos, culturales, geográficos y científicos.

Concepción del medio ambiente. En el campo de formación "Exploración y comprensión del mundo natural y social" se pretende, entre otras cuestiones, que los estudiantes desarrollen la perspectiva de explorar y entender el entorno, mediante el acercamiento sistemático y gradual a los procesos sociales y fenómenos naturales; entre los que se encuentran los temas ambientales a nivel regional, nacional o mundial, conforme se avanza en los grados escolares. De acuerdo con la orientación de las asignaturas referidas al campo de formación, se espera que los estudiantes de educación secundaria, además de conocer diversos temas relacionados con el medio ambiente, desarrollen las competencias para la vida que les permitan obtener la sensibilidad hacia los acontecimientos y problemas ambientales de nuestro tiempo y la habilidad para identificar y descubrir problemas de este ámbito; como también las habilidades para idear métodos y medios para contribuir a resolver o reducir esos problemas. Así como desarrollar la conciencia de los problemas ambientales, y los valores y actitudes, para la protección y conservación del medio ambiente.

Los contenidos de educación ambiental. En educación secundaria, con las asignaturas de Ciencias, Geografía de México y del Mundo, Historia, Tecnología y la "Asignatura Estatal" se culmina el campo de formación "Exploración y comprensión del mundo natural y social". En el plan de estudios 2011, se describen cada una de las asignaturas de educación secundaria. De este Plan, se retoman algunos de los principales aspectos de las asignaturas anteriormente referidas.

En la asignatura de Ciencias, los estudiantes se acercan al estudio de los fenómenos de la naturaleza y de su vida personal de manera gradual y con explicaciones metódicas y complejas, y buscan construir habilidades y actitudes positivas asociadas a la ciencia. Así como favorecer la toma de decisiones responsables e informadas a favor de la salud y del ambiente. 
En la asignatura Geografía de México y del Mundo, se pretende que los alumnos logren asumirse como parte del espacio geográfico, valoren los componentes naturales de la superficie terrestre, la biodiversidad, la dinámica de la población mediante los componentes sociales y culturales, así como la desigualdad socioeconómica para fortalecer su participación de manera informada, reflexiva y crítica ante los problemas sociales, el cuidado del ambiente, la vulnerabilidad de la población y la calidad de vida en las escalas nacional y mundial.

En la asignatura de Historia, tiene como objeto de estudio a la sociedad. desde una perspectiva crítica e integral donde los procesos no están acabados Pretende que los estudiantes comprendan el mundo donde viven para ubicar y darle importancia a los acontecimientos de la vida diaria, y usar críticamente la información para convivir con plena conciencia ciudadana.

En la asignatura de Tecnología, a los estudiantes se les orienta al estudio de la técnica y sus procesos de cambio, considerando sus implicaciones en la sociedad y en la naturaleza. Entre otras cuestiones, se busca que sepan tomar decisiones de manera responsable en el uso y la creación de productos y procesos técnicos.

Una cuestión que es importante subrayar, es la incorporación en el plan de estudios de una "Asignatura Estatal", en la cual se pueden desarrollar temas con diversos contenidos referidos a la educación ambiental.

La SEP, a través de la Subsecretaría de Educación Básica, la Dirección General de Desarrollo Curricular y la Dirección de Enlace y Vinculación, conforme al acuerdo secretarial número 384 y los lineamientos nacionales 2009 para el diseño y elaboración de los programas de esta asignatura, establece la inclusión de alguno de los siguientes campos temáticos:

Campo temático 1. La historia, la geografía y/o patrimonio cultural y natural de la entidad;

Campo temático 2. El estudio de los temas que abordan en más de una asignatura: educación ambiental, formación en valores, y educación sexual y equidad de género;

Campo temático 3. Estrategias para que los alumnos enfrenten y superen problemas y situaciones de riesgo;

Campo temático 4. Estrategias que fortalecen las habilidades para el estudio y el aprendizaje;

Campo temático 5. Lengua y cultura indígena.

El diseño de cada "Asignatura Estatal" corresponde a la autoridad educativa de cada Estado y los equipos académicos designados para tal fin, quienes en coordinación con los directivos y profesores de educación secundaria, eligen el tema y los contenidos del programa; para ello, se deben de fundamentar en la información que se disponga de los problemas y necesidades locales. En la "Asignatura Estatal", se propone a los estudiantes la oportunidad para integrar y aplicar aprendizajes del entorno social, cultural y natural; así como fortalecer los contenidos específicos de la región y la entidad, y apoyar el desarrollo del perfil de egreso de la Educación Básica y de las competencias para la vida, mediante el trabajo con situaciones y problemas particulares de la localidad, y el contexto donde viven y estudian.

En el ciclo escolar 2012-2013 se aprobaron 16 programas referidos a la educación ambiental en los siguientes estados: Aguascalientes, Baja California Sur, Campeche, Durango, Guerrero, Hidalgo, Jalisco, Estado de México, Michoacán, Morelos, Querétaro, Quintana Roo, Tabasco, Tlaxcala, Veracruz y Zacatecas.

Por otra parte, es necesario subrayar que, si los profesores no consideran relevante el abordar a la educación ambiental como contenido transversal, esta no es considerada. Los profesores tienen un papel fundamental para desarrollar los contenidos referidos a las relaciones 
del ser humano con el medio ambiente, en donde pueden abordar, entre otras cuestiones, las acciones de prevención, adaptación y/o resiliencia ante los problemas ambientales. A pesar del reconocimiento de la importancia de este papel, los esfuerzos para incorporar la educación ambiental en la formación docente inicial, son incipientes.

La educación ambiental en el nivel básico, abarca estrategias que buscan propiciar en la familia y en la comunidad la promoción de acciones para el mejoramiento de las condiciones ambientales. La educación ambiental también puede centrarse en la investigación y la acción de los niños para que comprendan cómo las acciones de los individuos afectan al ambiente. De ambas maneras, estarán desarrollando un sentido de responsabilidad compartida y destrezas que les permitirán continuar implicados durante la edad adulta (HART, 2008). La educación ambiental en las escuelas de educación básica, solo puede ser una realidad cuando existan profesores preocupados por llevarla a cabo, y por supuesto, profesores con una sólida formación ambiental.

\section{Educación ambiental en la formación de docentes para el nivel medio básico}

En 1989, la Secretaria de Desarrollo Urbano y Ecología (SEDUE) dio a conocer un diagnóstico para la educación normal, con resultados poco favorables en cuanto a la incorporación de la dimensión ambiental en su plan de estudios. Después de 25 años, los resultados de este diagnóstico, todavía son vigentes.

En el conjunto de instituciones de educación normal, en el ciclo escolar 2013-2014, se encontraban inscritos 132.205 en este nivel educativo (2014). Jóvenes que en el futuro ejercerán la docencia en las escuelas de educación básica. En este documento, solo se analizan al plan de estudios de educación normal superior (2012) que corresponde a la formación de profesores de educación secundaria.

Plan de estudios 2012. El acuerdo sectorial 269 establece que los contenidos básicos de los programas de estudio y orientaciones académicos del plan de estudios para la formación de profesores de educación secundaria (2012), en cada una de sus especialidades: Español, Matemáticas, Biología, Física, Química, Historia, Geografía, Formación Cívica y Ética, Lengua extranjera (Inglés o Francés) y Telesecundaria serán determinados por la SEP. El plan de estudios de la formación docente, se analiza considerando los aspectos identificados en el plan de estudios del nivel medio básico.

Transversalidad. La educación ambiental como un campo transversal está ausente, no se hace mención de los campos transversales. El plan de estudios enfatiza el desarrollo de competencias didácticas para que los futuros docentes logren la articulación y la secuencia de los contenidos educativos; tampoco se incluye a la educación ambiental en los campos de formación complementaria; se margina el abordaje interdisciplinario o multidisciplinario de los temas ambientales.

Origen de la problemática ambiental. Entre los aspectos propuestos en el perfil de egreso del futuro profesor de educación secundaria, se menciona que debe asumir y promover el uso racional de los recursos naturales y ser capaz de enseñar a los alumnos a actuar personal y colectivamente con el objetivo de proteger el ambiente. No se contemplan contenidos para comprender el origen de los problemas ambientales. Se observa una simplificación de los problemas ambientales y se centran en los referentes a los recursos naturales.

Concepción del conocimiento. En el plan de estudios se privilegia la formación adquirida en las disciplinas, por medio de las cuales se aprenderán contenidos científicos que aseguren el 
dominio de la disciplina que enseñará. En este plan de estudios no se considera los conocimientos de la pedagogía ambiental, se naturalizan los problemas ambientales, sin identificar las causas e implicaciones sociales de los problemas ambientales.

Concepción del medio ambiente. En el plan de estudios se hace mención de la importancia de que los futuros docentes aprecien y respeten la diversidad regional, social, cultural y étnica del país. El medio ambiente se interpreta como el escenario donde se desarrollan los grupos humanos, sin embargo, no aparece un concepto o una definición específica, sino conlleva a que cada futuro docente haga su propia interpretación.

Contenidos de educación ambiental. En el plan de estudios anterior (1999), en varios cursos se consideraron los contenidos relacionados con el medio ambiente. En la asignatura de educación ambiental y para la salud (2000), se consideró obligatoria para la Licenciatura en Biología y opcional en las licenciaturas de Física y Química. Esta asignatura tiene, entre otros objetivos, la concienciación de los estudiantes y el fortalecimiento de conocimientos, valores y competencias para actuar de manera individual y colectiva a favor del ambiente.

Además, se incluyó la asignatura opcional Educación ambiental (SEP, 2000) en la Licenciatura de Biología, cuyo propósito fue ampliar los conocimientos y las habilidades para analizar las situaciones ambientales y guiar a sus alumnos en la construcción de conocimientos y la formación de actitudes y valores que les permitan tomar decisiones tendientes a mejorar su relación el ambiente.

En el tercer semestre, se impartía la materia "Procesos vitales: la estructura y las funciones de los seres vivos"; en el quinto semestre: "Los seres vivos y su ambiente: la ecología"; y en el sexto semestre: "Educación ambiental y para la salud."

Estos cursos ya no fueron tomados en cuenta en el nuevo plan de estudios. El plan de estudios 2012 comprende cursos de formación general, de formación común y de formación especializada; en los cursos de los dos primeros campos no se encuentra alguna asignatura referida a la educación ambiental; los cursos de formación especializada de acuerdo al colectivo de docentes que trabajan determinadas especialidades, diseñaron los programas de estudio correspondientes para el sexto, el séptimo y el octavo semestre. Es de esperarse que alguno de estos, incluyan temas o contenidos referidos a la educación ambiental en el ciclo escolar 2015-2016.

\section{A modo de reflexiones finales}

A partir de los resultados de esta revisión teórica, se hace necesario cuestionar si la formación docente inicial en México está propiciando en los futuros docentes un interés por colaborar en la solución de los problemas y situaciones que viven los alumnos, así como las comunidades que rodean a las instituciones escolares. Como también, es necesario cuestionar si a los futuros docentes se les prepara para enfrentar los sistemas creados en los últimos años para el acceso a los puestos de trabajo; y sí a los estudiantes de las diferentes instituciones de educación superior que también aspiran a ejercer la docencia en las escuelas secundarias, adquieren una formación pedagógica para abordar la educación ambiental. Cuestionamientos que pueden generar nuevas rutas de investigación.

Los cambios en la educación ambiental y en la educación en general pueden parecer impresionantes cuando se prescriben desde las políticas educativas y los discursos que acompañan y fundamentan los planes y los programas, sin embargo, mientras no se introduzcan en el centro de los saberes y las prácticas cotidianas de los profesores, estos cambios se muestran de manera 
superflua y sobrepuesta de manera que aunque se generen propuestas de formación en relación a la educación ambiental se requiere ir más allá en las modalidades de formación que se proponen. ... ya ni los mejores programas de estudios ni el mejor material pedagógico podrán tener el efecto deseado si quienes están a cargo de los mismos, no han asimilado los objetivos de la EA y no son capaces de llevar a cabo el aprendizaje y las experiencias que la misma comporta, ni de utilizar eficazmente el material que tienen a su disposición (UNESCO, 1978, sp.).

La educación ambiental en la formación del profesorado contempla la articulación entre los conocimientos pedagógicos, científicos y ambientales (MATOS, 2011), propiciando las competencias para que puedan, no solo trasmitir la información ambiental, sino que puedan educar ambientalmente a sus alumnos. En este sentido, la educación ambiental requiere de profesores que se propongan lograr la autogestión de sus alumnos ante los problemas ambientales. Para ello, formar al futuro profesor de tal manera que facilite que sean sus alumnos quienes busquen la respuesta a los problemas que posibilite que aprendan a pensar por ellos mismos (GARCÍA, 2004, 172).

En las instituciones de educación normal como instituciones formadoras de docentes, se requiere de:

...promover iniciativas que aseguren una formación ambiental rigurosa de los docentes, así como el establecimiento de nexos obligados entre la formación inicial y la permanente para superar la dicotomía teoría-práctica, de tal forma que la primera sea el punto de arranque de la práctica docente, estableciendo procesos continuos de reflexión-acción-reflexión (Aramburu, 2000, 34).

Al revisar los contenidos del plan de estudios de la educación secundaria de la Reforma Integral de Educación Básica (RIEB) y el plan de estudios para la formación de profesores en educación secundaria de la Reforma Curricular de la Educación Normal (RCEN), se observan diferencias significativas.

En la formación inicial de los docentes se prioriza el dominio de los contenidos disciplinarios sobre los contenidos transversales, no se contemplan contenidos con un fuerte componente actitudinal y valoral, referidos al propio ser humano, de la sociedad y el ambiente natural. Se continúa así en el currículum, un abordaje tradicional, de forma aislada y circunstancial de los temas ambientales. La problemática ambiental no es tomada en cuenta como un tema relevante en el plan de estudios. Al respecto, es importante subrayar que:

...es necesario establecer una preparación que proporcione a los futuros docentes conocimientos significativos que genere una actitud, que conduzca a valorar la necesidad de una actualización permanente en función de los cambios y problemas que se producen local y globalmente (PEZA, 2011, 64).

Una valoración objetiva de los alcances de la RCEN, lleva a cuestionar la ausencia de la educación ambiental como campo transversal en la formación docente inicial.

La educación ambiental en la formación de docentes, no implica solo la comprobación y validación de los discursos teóricos ni las prácticas condensadas, sino el desarrollo de competencias ambientales que les permitan a los futuros docentes, incidir significativamente en la realidad de los problemas ambientales.

En la formación de docente inicial, los campos transversales se vuelven invisibles en la malla curricular y no son abordados en todos y cada uno de los espacios (principalmente en términos metodológicos). En el diseño del marco curricular de los nuevos programas de estudio, han de ser tomadas en cuenta no solo las propuestas de los especialistas, sino también, la 
experiencia y propuestas teóricas de los formadores de docentes. Una alternativa factible es propuesta por Pedroza y Argüello (2012), quienes argumentan que no se trata de incorporar a los planes de estudios, asignaturas relacionadas con la cuestión ambiental, sino más bien la necesidad de unificar un conocimiento en relación con todas las materias de manera sistémica.

En este marco, las instituciones formadoras de docentes han de considerar el desarrollo de proyectos de investigación educativa, como una de sus tareas prioritarias para retroalimentar sus programas de estudio.

En el campo de la educación ambiental, hay una incipiente línea de investigación referida a la formación de docentes en educación ambiental, entre los que se encuentran, Alcira y Perales (2006), Calixto (2009), Castañeda (2010), Peza (2011) y Perales (2010); se requieren proyectos de investigación en las instituciones formadoras de docentes, ya que sus resultados permitirán identificar y comprender los problemas que enfrentan los formadores de docentes, así como proponer alternativas para fomentar el desarrollo de habilidades docentes que promuevan una reforma en los planes de estudio del nivel medio básico es insuficiente e ineficiente, si no se empata con la reforma del plan de estudios para la formación de docentes. ¿Cómo van a tratar los contenidos ambientales los docentes, si ellos carecen de una formación en educación ambiental? Se ha de tomar en cuenta la dinámica de las propias instituciones, los formadores de docentes y las corrientes del pensamiento del quehacer docente (ZEICHNER, 1983).

En este sentido, emergen varios retos en la formación docente en educación ambiental:

- desarrollar propuestas tendientes a la ambientalización curricular;

- capacitar/formar a los docentes;

- generar proyectos, programas y estrategias;

- fomentar una cultura ambiental;

- contextualizar la práctica, vinculando los problemas ambientales locales con los globales; y

- vincular la pedagogía con diversas disciplinas ambientales.

La formación docente, así como su participación en el cambio educativo es esencial para que puedan tener éxito las propuestas, los programas y las inquietudes y necesidades de los alumnos, sobre todo si se trata de cambios complejos como es el caso de la educación ambiental que requiere de procesos complejos y metas tanto a corto plazo como a largo plazo. No basta con que los profesores adquieran nuevos conocimientos sobre los contenidos curriculares o nuevas técnicas de enseñanza, los profesores no son simples aprendices técnicos, sino también son aprendices sociales (HARGREAVES, 2003).

Se hace necesario en la formación de profesores, tomar en cuenta otros elementos como el deseo, la conciencia y/o la convicción de querer hacer el cambio en este caso, el cambio relacionado con la formación de una conciencia de ciudadanía común (MORENO y BIANCHINI, 2015).

Las escuelas formadoras de docentes, pueden constituirse en las instituciones que impulsen a los educandos a mirarse como parte de una ciudadanía, favorecer la comprensión integral del medio ambiente y promover la actuación fundamentada y crítica ante los problemas ambientales.

\section{Referencias}

ALCIRA, M.; PERALES, F. J. La resolución de problemas ambientales en la escuela y en la formación inicial de maestros. Revista iberoamericana de educación, Madrid, n. 40, p. 111-124. Disponible en: http://www.rieoei.org/rie40a06.htm. Acceso el: 6 enero 2015. 
ARAMBURU, F. Medio ambiente y educación, Madrid: Síntesis, 2000.

BARBER, M.; MORUSHED, M. How the world's best-performing school systems come out on top. London: McKinsey \& Company, Social Sector Office, 2007.

CALIXTO, R. Representaciones sociales del medio ambiente. México: UPN, 2009.

CASTAÑEDA, O. Perspectiva docente sobre la educación ambiental. Revista Casa del Tiempo, Ciudad de México, n. 29, p. 80-85, 2010.

DE AMBROSIO, M. Todo lo que necesitas saber sobre el cambio climático. Buenos Aires: Paidós, 2014. GARCÍA, E. Educación ambiental, constructivismo y complejidad. España: Díada Editorial, 2004.

GARCÍA, J. De la transversalidad a la Agenda 21, en el V Congreso Iberoamericano de Educación Ambiental. México: Programa de las Naciones Unidas para el Medio Ambiente, 2007.

GARCÍA R. Sistemas complejos: conceptos, métodos y fundamentación epistemológica de la investigación interdisciplinaria. México: Gedisa, 2013.

GASPARETTO, I.; ZATTONI, M; PROTTI, F. Educação Ambiental em contextos não escolares: definindo, problematizando e exemplificando. Pesquisa em Educação Ambiental, Ribeirão Preto, v. 7, n. 2, p. 119-131, 2012

GONZÁLES GAUDIANO, E. Educación ambiental, Historia y conceptos: a veinte años de Tbilisi. México, Sistemas Técnicos de Edición, 1997

1998

Centro y periferia de la educación ambiental: un enfoque antiesencialista. México: MundiPrensa,

La educación ambiental en México: logros, perspectivas y retos de cara al nuevo milenio. In: Memoria de educación ambiental para un desarrollo sustentable, taller de especialistas y 20 . Foro Nacional. México: Semarnat, Anea, Cesu, Universidad Autónoma de Aguascalientes, p.15-46, 2002.

La investigación en educación ambiental en México: entre Sísifo y Pigmalión. In: CONGRESO NACIONAL DE INVESTIGACIÓN EN EDUCACIÓN AMBIENTAL, 2., 2011, Puebla de Zaragoza. Anais... Puebla de Zaragoza: Benemérita Universidad Autónoma de Puebla, 2011. Disponible en: http://edgargonzalezgaudiano.blogspot.mx/2011/03/la-investigacion-en-educacion-ambiental.html. Acceso el: 6 enero 2016.

GRUPO INTERGUBERNAMENTAL DE EXPERTOS SOBRE EL CAMBIO CLIMÁTICO. Cambio climático 2013. Bases física. Resumen para responsables de políticas. Santiago: OMN; PNUMA. Disponible en: https://www.ipcc.ch/pdf/assessment-report/ar5/wg1/WG1_AR5_SPM_ES.pdf .Acceso el: 6 jun. 2015.

GUIMARÃES, J. M. M.; ALVEZ, J. M. Formação de professores na área de Educação Ambiental: uma análise dos anais da ANPEd (2009-2011). Pesquisa em Educação Ambiental, Ribeirão Preto, v. 7, n. 1, p. 49-66, 2012.

HARGREAVES, A. Profesorado, cultura y posmodernidad, cambian los tiempos, cambia el profesorado. Madrid: Morata, 2003.

HART, R. Children's participation: the theory and practice of involving young citizens in community development and environmental care. London: Unicef, 2008.

LOVELOCK, J. La venganza de la Tierra. Barcelona: Editorial Planeta, 2007. 
MATOS, M. A formação de professores/as e de educadores/as ambientais: aproximações e distanciamentos. Pesquisa em Educação Ambiental, Ribeirão Preto, v. 4, n. 2, p. 203-214. 2009. Disponible en: <http://www.revistasusp.sibi.usp.br/pdf/pea/v4n2/14.pdf>. Acceso el: 7 feb. 2016.

La formación de profesores (as) y de educadores (as) ambientales: acercamientos y distanciamientos. In: CALIXTO, R. (Coord.). Experiencias latinoamericanas en educación ambiental. México: CECyTE NL-CAEIP, 2011. p. 59-76.

MIRANDA, F. La reforma curricular en la Educación Básica. In: ARNAUT, A.; GIORGULI, S. (Coord.). Los grandes problemas en México. VII. Educación. México: El Colegio de México, 2012.

MORENO, O.; BIANCHINI, D. Interrelación local-global y ciudadanía planetaria: una cuestión importante para la educación del siglo XXI. Pesquisa em Educação Ambiental, Ribeirão Preto, v. 10, n. 1, p. 43-56, 2015.

PEDROZA, R.; ARGÜELLO, F. Interdisciplinariedad y transdisciplinariedad en los modelos de enseñanza de la cuestión ambiental. Cinta moebio 15, p. 286-299, 2012.

PERALES, F. J. Cambios en la percepción ambiental de los futuros maestros de Educación Primaria. In: CONGRÉS INTERNACIONAL DE DIDACTIQUES, 2., 2010, Girona, Anais... Girona: Universitat de Girona, 2010. Disponible en: http://hdl.handle.net/10256/2699. Acceso el: 21 dic. 2015.

PEZA, G. La educación ambiental en el espacio social de los estudiantes normalistas. El estudio de caso en las Normales de Nuevo León. Transatlántica de educación, año VI, v. 9, n. 30, p. 62-66, 2011.

RANDERS, J. 2052: a global forecast for the next forty years. Chelsea: Green Publishing, 2012.

SECRETARÍA DE EDUCACIÓN PÚBLICA. Acuerdo 269. México: Diario Oficial de la Federación, 2000. . Educación básica. Secundaria. Asignatura Estatal. Lineamientos Nacionales. México: SEP, 2006.

Disponible Catálogo Nacional 2012. Programas de estudio de asignatura estatal. Plan de estudios 2011. http://basica.sep.gob.mx/reformasecundaria/asignaturaestatal/PresentacionRelacionCatalogo2012.pdf. Acceso el: 21 oct. 2015.

. Sistema educativo de los Estados Unidos Mexicanos, principales cifras, ciclo escolar 2013-2014. México: SEP, 2014.

SEDUE. Recomendaciones para la incorporación de la dimensión ambiental en el sistema educativo nacional. México: Sedue, 1989.

SEMARNAT. Estrategia de educación ambiental para la sustentabilidad en México. México: Semarnat, 2006.

UNESCO. Conferencia Intergubernamental sobre Educación Ambiental. Tiblisi (URSS), Paris: Unesco, 1978.

. La declaración de Ahmedabad: una llamada a la acción. Paris: Unesco, 2007

ZEICHNER, K. M. Alternative Paradigms of Teacher Education. Journal of Teacher Education, Thousand Oaks, v. XXXIV, n. 3, p. 3-9, 1983. 